Pacific Journal of Mathematics

EXTERNAL VISIBILITY

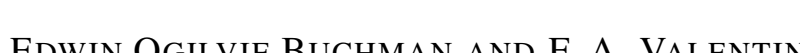




\section{EXTERNAL VISIBILITY}

\section{Edwin Buchman and F. A. Valentine}

It is possible to see any eleven vertices of an opaque solid regular icosahedron from some appropriate point, although it is not possible to see all twelve vertices simultaneously. In this paper we refer to visibility in the complement of a convex set as external visibility. Valentine has investigated external visibility properties in Euclidean space $E^{2}$ and $E^{r}$. One question raised was the following: does there exist a fixed number $h$ such that if every $h$ vertices of an arbitrary bounded closed convex polyhedron in $E^{3}$ can see some common point externally, then all the vertices can see some common point externally?

The answer is no, which is surprising since the corresponding question in $E^{2}$ will be answered affirmatively, with $h=5$. Figure 2 illustrates a solid convex polyhedron with $4 n$ vertices $(n=4$ in the illustration), where the top and bottom vertices $\left(V_{1}\right.$ and $\left.V_{2}\right)$ have valence $2 n-1$. Each collection of $4 n-1$ vertices of this polyhedron can see some point in space externally, yet there is no point which all $4 n$ vertices can see externally simultaneously. It will be shown that this polyhedron can be constructed for arbitrarily large $n$.

1. Definitions and notation. The boundary and interior of a set $S$ are designated by bd $S$ and int $S$ respectively. The closed line segment joining the points $x$ and $y$ is $x y$, and the line through $x$ and $y$ is $L(x, y)$.

In order to state theorems on external visibility precisely, we define several kinds of external visibility properties:

Definition 1. Suppose $K$ is a closed convex set in $E^{r}$, and suppose $x \in \operatorname{bd} K$ and $p \in E^{r}$. Let $R$ be a ray emanating from the origin of $E^{r}$, and let $R_{x}$ be the ray from $x$ in the direction of $R$. Then

(a) $x$ and $p$ see each other externally relative to $K$ if $x p$ intersects $K$ only at $x$ (i.e., $x p \cap K=x$ ).

(b) $x$ and $p$ see each other externally relative to int $K$ if $x p$ misses int $K$ (i.e., $x p \cap$ int $K=\varnothing$ ).

(c) a set $S \subset \mathrm{bd} K$ and $p$ see each other externally relative to $K$ (int $K$ ) if all points of $S$ see $p$ externally relative to $K$ (int $K$ ).

(d) $x$ sees $\infty$ externally relative to $K$ in the direction of $R$ if the ray $R_{x}$ intersects $K$ only at $x$ (i.e., $R_{x} \cap K=x$ ).

(e) $x$ sees $\infty$ externally relative to int $K$ in the direction of $R$ if the ray $R_{x}$ misses int $K$ (i.e., $R_{x} \cap$ int $K=\varnothing$ ). 
(f) a set $S \subset$ bd $K$ sees $\infty$ externally relative to $K$ (int $K$ ) in the direction of $R$ if all points of $S$ see $\infty$ in the direction of $R$ externally relative to $K$ (int $K$ ).

Definition 2. A convex body in $E^{r}$ is a bounded closed convex set with a nonempty interior.

In this paper, $K$ will always be a convex body in $E^{r}$.

\section{External visibility in $E^{2}$.}

THEOREM 1. Let $K$ be a convex body in $E^{2}$ and suppose $S$ is a closed subset of bd $K$. If every five points of $S$ can see some common point externally relative to int $K$ then all of $S$ can see some common point externally relative to $K$.

Proof. Since $K$ is a bounded closed convex set in $E^{2}$, it is easy to see that every five points of $S$ can see a common point externally relative to $K$. Hence, we can assume this stronger condition. Since the theorem is trivial if $S$ contains fewer than six points, let $x_{i}(i=1,2,3,4,5,6)$ be six points of $S$ chosen in a clockwise sense on the boundary of $K$. The exterior angle $\alpha_{2}$ at $x_{2}$ is determined as in Figure 1, so that it is the angle between the two rays $\overrightarrow{x_{1} x_{2}}$ and $\overrightarrow{x_{2} x_{3}}$, and this definition extends cyclically.

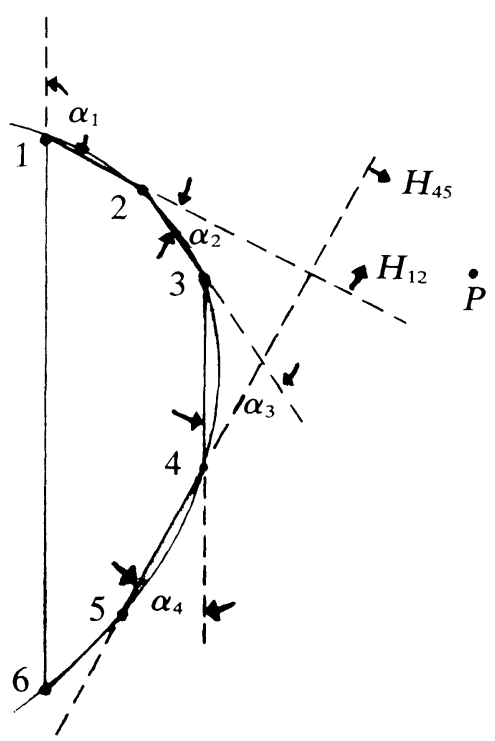

FIG. 1 
Since $\sum_{i=1}^{6} \alpha_{i}=2 \pi$, there must be some three consecutive $x_{i}$, say $x_{2}, x_{3}, x_{4}$ (without loss of generality), such that

$$
\alpha_{2}+\alpha_{3}+\alpha_{4} \leqq \pi
$$

Now $p$ exists by hypothesis so that $p$ can see $x_{1}, x_{2}, x_{4}, x_{5}, x_{6}$ externally relative to $K$. We claim that $p$ can likewise see $x_{3}$. To prove this, assume that $p$ does not see $x_{3}$ externally relative to $K$. Since the subset of bd $K$ which $p$ can see is always a connected arc, it must be that $p$ sees the entire arc of bd $K$ which connects $x_{1}$ and $x_{2}$ and does not contain $x_{3}$. This implies that $p$ is in the open half plane $H_{12}$ bounded by $L\left(x_{1}, x_{2}\right)$ which does not contain $x_{3}$. Also $p$ must be in $H_{45}$, correspondingly defined. By the inequality (1), $H_{12} \cap H_{45} \neq \varnothing$ and lies on the same side of $L\left(x_{1}, x_{5}\right)$ as $x_{3}$, and (1), in fact, holds as a strict inequality. Since $p$ sees $x_{1}$ and $x_{5}$, and therefore some connected arc of bd $K$ connecting $x_{1}$ and $x_{5}$, externally relative to $K$, the preceding sentence implies that $p$ sees $x_{3}$ externally relative to $K$, contrary to our assumption that such was not true. Since the $x_{i}$ were arbitrarily chosen in $S$, we have shown that every six points of $S$ can see some point $p$ externally relative to $K$.

We can repeat this procedure to show that every seven points of $S$ can see some common point externally relative to $K$. Let $x_{i}$ ( $i=$ $1, \cdots, 7)$ be seven arbitrary points of $S$, enumerated in a clockwise sense around bd $K$. We define the angles $\alpha_{i}$ as before, and find some three which satisfy (1). Using the result established above, we can find a point $p$ which can see $x_{1}, x_{2}, x_{4}, x_{5}, x_{6}, x_{7}$ externally relative to $K$. The argument of the preceding paragraph using $H_{12}$ and $H_{45}$ shows that $p$ can see all seven of the $x_{i}$ externally relative to $K$. Since this procedure can be repeated for $8,9,10, \cdots$ points of $S$, it follows by induction that every finite collection of points of $S$ can see some common point externally relative to $K$.

This last fact implies that every finite number of points of $S$ can see $\infty$ in some direction externally relative to the interior of $K$. If these directions are mapped onto the unit circle relative to its center, then the image of the set of such directions for each point of $S$ is a closed arc of the unit circle. Since each finite collection of such arcs has a nonempty intersection, then the usual compactness argument implies that there exists a direction such that $S$ can see $\infty$ in this direction externally relative to int $K$. Therefore $S$ lies on a closed arc of bd $K$ lying between two parallel lines of support to bd $K$. Because $S$ is closed, if we take $C$ to be the minimal arc containing $S$ between the two parallel lines of support, then the endpoints of $C$ belong to $S$. Applying the visibility hypothesis to the endpoints of $C$ and any third point of $S$ between the parallel lines, it is not difficult to find a point which can see all of $S$ externally relative to $K$. This completes the proof of Theorem 1 . 
The proofs of the following two theorems are very similar to the proof of Theorem 1 .

THEOREM 2. Let $K$ be a convex body in $E^{2}$ and suppose $S$ is a closed subset of bd $K$. If every five points of $S$ can see $\infty$ in some common direction externally relative to $K$, then there exists some fixed direction in which all of $S$ can see $\infty$ externally relative to $K$.

Proof. In the proof of Theorem 1, replace the point $p$ with a ray $R$ emanating from the origin. We modify the argument so that for each point $x \in \mathrm{bd} K$ the property of seeing $p$ externally relative to $K$ is replaced by seeing $\infty$ in the direction of $R$ externally relative to $K$. Also, instead of the condition $p \in H_{12} \cap H_{45}$, we conclude that the ray emanating from $x_{3}$ in the direction of $R$ intersects $H_{12} \cap H_{45}$. Thus modified, the first two paragraphs of the proof show that every finite subset of $S$ can see $\infty$ in some common direction externally relative to $K$. The third paragraph, unchanged except for the final conclusion, completes the argument.

THEOREM 3. Let $K$ be a convex body in $E^{2}$ and suppose $S$ is a subset of bd $K$. If every six points of $S$ can see $\infty$ in some common direction externally relative to int $K$, then there exists some fixed direction in which all of $S$ can see $\infty$ externally relative to int $K$.

Proof. Suppose that $S$ contains at least seven points $x_{i}(i=$ $1, \cdots, 7)$. Using the notation of Figure 1 for the corresponding angles $\alpha_{i}$, we have $\sum_{i=1}^{7} \alpha_{i}=2 \pi$, so there must be some three consecutive $x_{i}$, say $x_{2}, x_{3}, x_{4}$ (without loss of generality), such that $\alpha_{2}+\alpha_{3}+\alpha_{4}<\pi$. (This is (1) rewritten as a strict inequality). Let $R$ be a ray chosen by hypothesis so that $x_{i}(i=1,2,4,5,6,7)$ each can see $\infty$ in the direction of $R$ externally relative to int $K$. The strict inequality just stated implies that $H_{12}$ and $H_{45}$ have a nonempty intersection, and intersect on the same side of $L\left(x_{1}, x_{5}\right)$ in which $x_{3}$ lies. Since $x_{1}, x_{2}, x_{4}, x_{5}$ all can see $\infty$ in the direction of $R$ externally relative to int $K$, this implies that $x_{3}$ too can see $\infty$ in the direction of $R$. By an argument similar to that for Theorem 1 all seven of the $x_{i}$ can see $\infty$ in the same direction externally relative to int $K$. As in Theorems 1 and 2, we proceed by induction to show that every finite subset of $S$ can see $\infty$ in some direction externally relative to int $K$. Finally, we need only the first portion of the last paragraph of the proof of Theorem 1 to complete the proof, whether $S$ is closed or not.

Counterexamples. The number five in Theorems 1 and 2, and the number six in Theorem 3 are best in each case, as is shown by taking $K$ to 
be a regular pentagon in Theorems 1 and 2, and a regular hexagon in Theorem 3, and taking $S$ in each case to be the set of vertices of $K$.

To show that $S$ must be closed in Theorems 1 and 2, let $K$ be the closed convex set which is the union of a square region and a semicircular region where the diameter of the semicircle is an edge of the square. Hence, $K$ looks like a Norman window. Let $S$ be an arc of bd $K$ consisting of the open semicircular arc of bd $K$ plus one of the adjacent closed edges of the square. Although every finite subset of $S$ can see a common point (and also $\infty$ in a common direction) externally relative to $K$ (and hence also relative to int $K$ ), no point exists which all of $S$ can see externally relative to $K$ or to int $K$, and no direction exists in which $S$ can see $\infty$ externally relative to $K$. It should be noted that $S$ can see $\infty$ externally relative to int $K$ in one direction, so the conclusion of Theorem 3 cannot be strengthened by replacing "relative to int $K$ " by "relative to $K$ ".

3. External visibility in $E^{3}$. In this section we construct a closed convex polyhedron $K$ having $4 n$ vertices $(n \geqq 3)$ with the property that for each $4 n-1$ of its vertices some common point of external visibility relative to $K$ can be found, yet there is no point which all $4 n$ vertices can see simultaneously externally relative to $K$. This shows that no Helly number can be found for external visibility relative to all convex bodies $K$ in $E^{3}$, that is, there is no fixed integer $h$ such that for each $K$ and each closed set $S \subset$ bd $K$ if every $h$ points of $S$ can see some common point externally relative to $K$, then all of $S$ can see some common point externally relative to $K$.

Construction. Let $n$ be an integer with $n \geqq 3$, and let $d>0$. The points $V_{1}^{i}(i=1, \cdots, 2 n-1)$ are in the plane $z=d$ in $E^{3}$ and also are the vertices of a regular $(2 n-1)$-gon centered at $(0,0, d)$. Call this polygon $G_{1}$. Rotate $G_{1}$ about the $z$-axis through an angle $\pi /(2 n-1)$ and translate it parallel to the $z$-axis so that the center $(0,0, d)$ goes to $(0,0,-d)$. Call the new polygon $G_{2}$. The vertices of $G_{2}$ are $V_{2}^{i}(i=1, \cdots, 2 n-1)$ where $V_{2}^{1}$ is "between" $V_{1}^{1}$ and $V_{1}^{2}$ in terms of the rotation about the $z$-axis, and the remaining vertices $V_{2}^{i}$ advance cyclically. This is illustrated in Figure 2 when $n=4$.

We can find a point $V_{1}=(0,0, h)$ on the $z$-axis such that $V_{1}$ is coplanar with $V_{1}^{1}, V_{2}^{1}$, and $V_{1}^{2}$, and this coplanar property continues to hold if the superscripts are cyclically advanced. Correspondingly we can choose $V_{2}=(0,0,-h)$ so that it will have the same coplanar properties with $V_{2}^{1}, V_{1}^{2}$, and $V_{2}^{2}$, etc.

The polyhedron $K$ which has the $4 n$ vertices $V_{1}, V_{2}, V_{1}^{i}, V_{2}^{i},(i=$ $1, \cdots, 2 n-1)$ has $4 n-2$ congruent quadrilateral faces, and the entire surface is the union of two congruent conical surfaces which have 


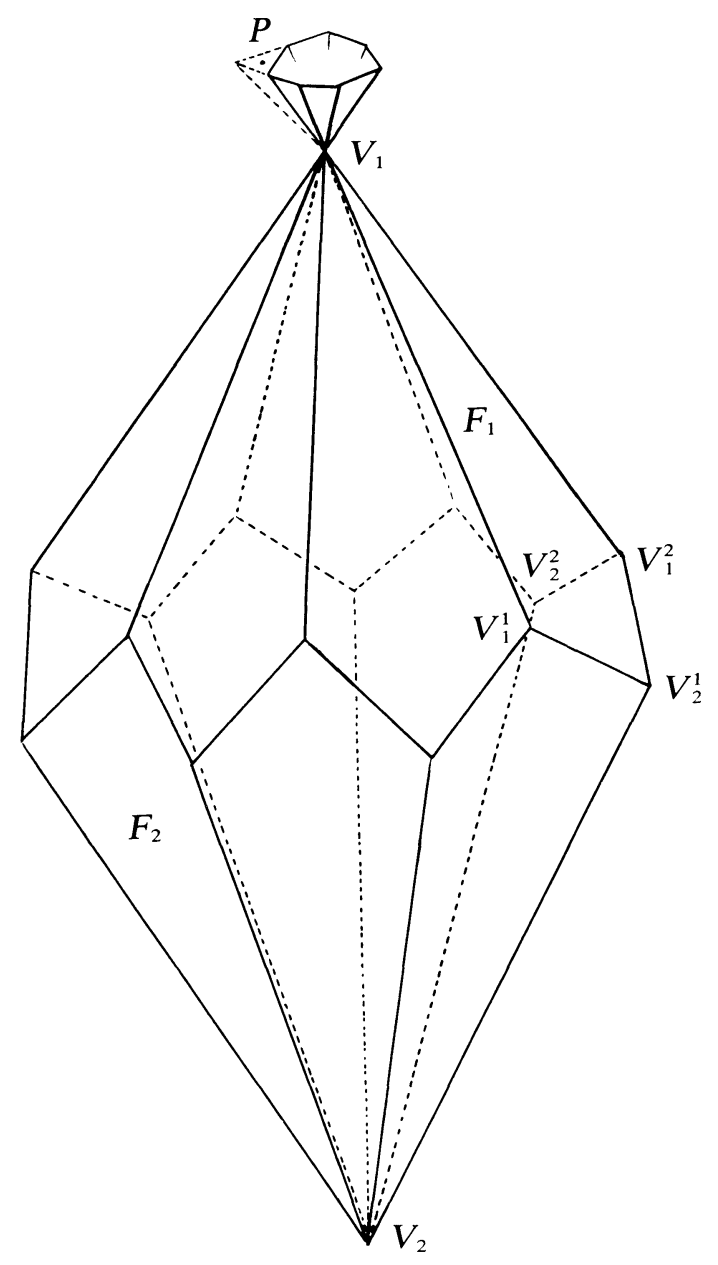

FIG. 2

opposite orientation relative to the $z$-axis and which join along a serrated zig-zag path, somewhat like a Japanese lantern without pleats. (See Figure 2, where some of the vertical dimensions have been exaggerated for clarity).

Visibility properties of $K$. In the following, we refer to external visibility relative to $K$ simply as visibility. We claim first that if $M$ is any set of $4 n-1$ vertices of $K$, then there is some point in space which can see each vertex in $M$. Obviously, if the vertex excluded from $M$ is $V_{1}$, then an appropriate point of visibility for $M$ can be found on the $z$-axis by choosing $(0,0, u)$ where $u<-h$, and similarly for $V_{2}$. Hence assume that the vertex excluded from $M$ is $V_{2}^{1}$. (Because of symmetry our argument will hold for each of the remaining vertices). Let $F_{1}$ be the face 
of $K$ determined by $V_{1}^{1}, V_{2}^{1}, V_{1}^{2}$, and $V_{1}$. Consider the intersections of the plane $z=z_{0}>h$ with the planes of the faces of $K$ which pass through $V_{1}$. We can find a point $P$ in this plane, as in Figure 2, such that $P$ and int $K$ lie in the same open half space bounded by the plane of $F_{1}$, yet such that each of the other planes of the upper faces of $K$ strictly separates $P$ from int $K$. Thus $P$ can see every point on each of the upper quadrilateral faces of $K$ except $F_{1}$, and so $P$ can see every vertex of $K$ except for $V_{2}^{1}$ and possibly $V_{2}$. Now note that since the faces of $K$ are parallel in opposite pairs, there is some face $F_{2}$ containing $V_{2}$ which is parallel to $F_{1}$. We select a point $P^{\prime}$ on the ray $\overrightarrow{V_{1} P}$ sufficiently far from $V_{1}$ so that the plane of face $F_{2}$ strictly separates $P^{\prime}$ from int $K$. This point $P^{\prime}$ can see each vertex of $K$ except $V_{2}^{1}$.

There is no point which can see all of the vertices of $K$ simultaneously. To show this it is convenient to divide the space $E^{3}$ into three regions separated by the parallel planes $z=h$ and $z=-h$, where $h$ and $-h$ are the $z$ coordinates of $V_{1}$ and $V_{2}$ respectively. In the region $z>h$, consider a plane $z=z_{0}$, where $z_{0}$ is an arbitrary number greater than $h$. The planes of the faces of $K$ containing $V_{1}$ will intersect this plane in a $(2 n-1)$-gon. (In Figure 3, this is illustrated for $n=4$. The extensions of the sides of the heptagon thus obtained are not shown, but the point $P$ used earlier is shown for reference.) The planes of the faces which contain $V_{2}$ will intersect the plane $z=z_{0}$ in a larger $(2 n-1)$-gon with sides parallel to those of the first $(2 n-1)$-gon. Clearly any point inside the larger $(2 n-1)$-gon cannot see $V_{2}$. So let $Q$ be a point outside the larger $(2 n-1)$-gon. In this case a vertex $V_{2}^{\imath}$ exists such that $Q$ and $K$ lie on the same side of each of the three faces of $K$ which determine $V_{2}^{i}$, and hence $Q$ cannot see $V_{2}^{i}$. In Figure 3, the vertex $V_{2}^{i}$ is the intersection of the three planes indicated by the three arrows, and the point $Q$ illustrated cannot see $V_{2}^{i}$ because of the reasons stated above.

For points in the region $z<-h$, exactly the same type of argument as above applies. For points in the region $-h \leqq z \leqq h$, we take again a cross section $z=z_{0}$, where $-h \leqq z_{0} \leqq h$, and apply a similar type of reasoning, which differs very little from the preceding cases. This completes the proof.

It is interesting to note the following fact (we continue to refer to external visibility relative to $K$ simply as visibility). There exists a convex body $K$ in $E^{3}$ and a closed set $T \subset E^{3}$ containing $4 n$ points (where $n$ may be arbitrarily large) such that every $4 n-1$ points of $T$ can see a common point of bd $K$, yet all the points of $T$ cannot see a common point of bd $K$. To see this, let $K$ be the polyhedron constructed above. We have already seen that there exist $4 n$ points in $E^{3}$, each of which can see all but one of the vertices of $K$. If we take $T$ to be the set containing these $4 n$ points, this implies directly that every $4 n-1$ points of $T$ can see a common vertex of $K$. Yet if all of $T$ could see a common point of 


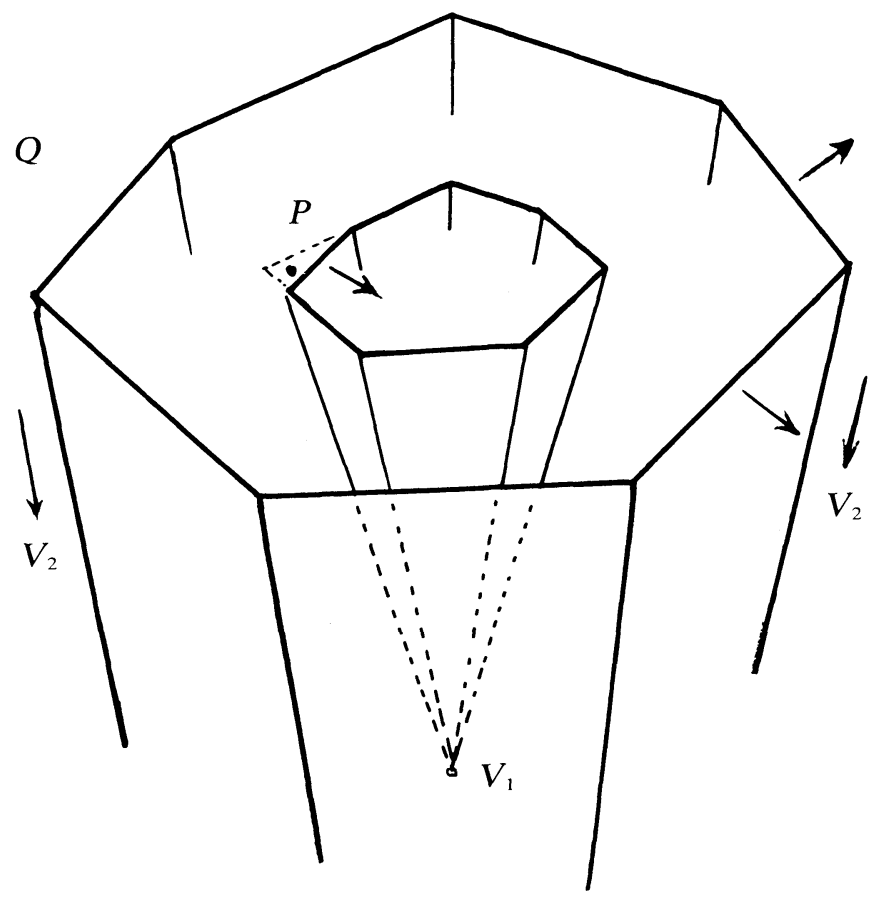

FIG. 3

bd $K$, then it is easy to see that all of $T$ could see some common vertex $V$ of $K$. But since some point of $T$, by construction of $T$, can see all the vertices of $K$ other than $V$, this point of $T$ could then see all the vertices of $K$. However, $K$ was constructed so that this is impossible. Hence, no Helly number exists for this type of external visibility in $E^{3}$, which answers a question raised in [1].

It is also worth mentioning that the essential properties which make the polyhedron $K$ a counterexample in $E^{3}$ can be extended to give corresponding counterexamples in $E^{r}$, where $r>3$. Define the convex body in $E^{r}$ to be the Cartesian product of $K$ with an appropriate number of copies of the interval $[-1,1]$, and define the set of points in bd $K$ as those whose first three coordinates correspond to vertices of $K$, and whose remaining coordinates are zero. (Note that these points are no longer vertices of the convex body.)

\section{REFERENCE}

1. F. A. Valentine, Visible shorelines, Amer. Math. Monthly, 77 (1970), 146-152.

Received February 27, 1976.

California State University, Fullerton 


\section{PACIFIC JOURNAL OF MATHEMATICS}

\section{EDITORS}

RICHARD ARENS (Managing Editor)

University of California

Los Angeles, California 90024

\author{
R. A. Beaumont \\ University of Washington \\ Seattle, Washington 98105
}

\section{J. DugunduI}

Department of Mathematics University of Southern California Los Angeles, California 90007

D. Gilbarg and J. Milgram

Stanford University

Stanford, California 94305

\section{ASSOCIATE EDITORS}

E. F. BECKENBACH
B. H. NeumanN

F. Wolp
K. YoshidA

\section{SUPPORTING INSTITUTIONS}

UNIVERSITY OF BRITISH COLUMBIA

CALIFORNIA INSTITUTE OF TECHNOLOGY

UNIVERSITY OF CALIFORNIA

MONTANA STATE UNIVERSITY

UNIVERSITY OF NEVADA

NEW MEXICO STATE UNIVERSITY

OREGON STATE UNIVERSITY

UNIVERSITY OF OREGON

OSAKA UNIVERSITY

\author{
UNIVERSITY OF SOUTHERN CALIFORNIA \\ STANFORD UNIVERSITY \\ UNIVERSITY OF HAWAII \\ UNIVERSITY OF TOKYO \\ UNIVERSITY OF UTAH \\ WASHINGTON STATE UNIVERSITY \\ UNIVERSITY OF WASHINGTON \\ AMERICAN MATHEMATICAL SOCIETY
}

The Supporting Institutions listed above contribute to the cost of publication of this Journal, but they are not owners or publishers and have no responsibility for its contents or policies.

Mathematical papers intended for publication in the Pacific Journal of Mathematics should be in typed form or offset-reproduced (not dittoed). double spaced with large margins. Underline Greek letters in red, German in green, and script in blue. The first paragraph or two must be capable of being used separately as a synopsis of the entire paper. Items of the biblography should not be cited there unless absolutely necessary, in which case they must be identified by author and Journal, rather than by item number. Manuscripts, in duplicate, may be sent to any one of the four editors. Please classify according to the scheme of Math. Reviews, Index to Vol. 39. All other communications should be addressed to the managing editor, or Elaine Barth, University of California, Los Angeles, California, 90024.

100 reprints are provided free for each article, only if page charges have been substantially paid. Additional copies may be obtained at cost in multiples of 50 .

The Pacific Journal of Mathematics is issued monthly as of January 1966. Regular subscription rate: $\$ 72.00$ a year (6 Vols., 12 issues). Special rate: $\$ 36.00$ a year to individual members of supporting institutions.

Subscriptions, orders for back numbers, and changes of address should be sent to Pacific Journal of Mathematics, 103 Highland Boulevard, Berkeley, California, 94708.

PUBLISHED BY PACIFIC JOURNAL OF MATHEMATICS, A NON-PROFIT CORPORATION Printed at Jerusalem Academic Press, POB 2390, Jerusalem, Israel.

Copyright (C) 1976 Pacific Journal of Mathematics All Rights Reserved 


\section{Pacific Journal of Mathematics}

\section{Vol. 64, No. $2 \quad$ June, 1976}

Richard Fairbanks Arnold and A. P. Morse, Plus and times............. 297

Edwin Ogilvie Buchman and F. A. Valentine, External visibility ......... 333

R. A. Czerwinski, Bonded quadratic division algebras.............. 341

William Richard Emerson, Averaging strongly subadditive set functions in unimodular amenable groups. II .........................

Lynn Harry Erbe, Existence of oscillatory solutions and asymptotic behavior for a class of third order linear differential equations ............. 369

Kenneth R. Goodearl, Power-cancellation of groups and modules........ 387

J. C. Hankins and Roy Martin Rakestraw, The extremal structure of locally compact convex sets ...................................

Burrell Washington Helton, The solution of a Stieltjes-Volterra integral

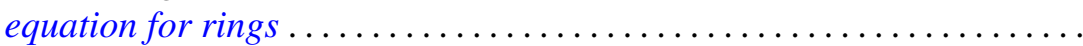

Frank Kwang-Ming Hwang and Shen Lin, Construction of 2-balanced

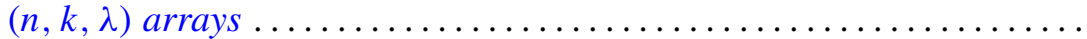

Wei-Eihn Kuan, Some results on normality of a graded ring ... 455

Dieter Landers and Lothar Rogge, Relations between convergence of series and convergence of sequences ......................... 465

Lawrence Louis Larmore and Robert David Rigdon, Enumerating immersions and embeddings of projective spaces ................

Douglas C. McMahon, On the role of an abelian phase group in relativized problems in topological dynamics..................

Robert Wilmer Miller, Finitely generated projective modules and TTF classes...

Yashaswini Deval Mittal, A class of isotropic covariance functions ...

Anthony G. Mucci, Another martingale convergence theorem ...

Joan Kathryn Plastiras, Quasitriangular operator algebras ...

John Robert Quine, Jr., The geometry of $p\left(S^{1}\right) \ldots \ldots \ldots$. 